\title{
Transcriptomes of microglia
} in the presence and absence of Type I Interferon signaling

\author{
Carlos Talavera-López ${ }^{1 \dagger}$, Barbara Capuccini ${ }^{1,2+}$, Richard Mitter ${ }^{4}$, Jing-wen Lin ${ }^{1,3}$ and Jean Langhorne ${ }^{1^{*}}$ (I)
}

\begin{abstract}
Objectives: Plasmodium berghei ANKA infection in mice is a model for human cerebral malaria, the most severe complication of Plasmodium falciparum infection. Responses of brain microglia have been little investigated, and may contribute to the pathogenesis of cerebral malaria. We showed previously that microglia are activated in $P$. berghei infections, and that Type 1-Interferon signaling is important for activation. This dataset compares transcriptomic profiles of brain microglia of infected mice in the presence and absence of Type 1 interferon signaling, with the aim of identifying genes in microglia in this pathway during experimental cerebral malaria.

Data description: We documented global gene expression from microglial RNA from uninfected and $P$ bergheiinfected wild-type C57BL/6 and IFNA Receptor Knock-out mice using Illumina Beadarrays. Principal component analysis showed 4 groups of samples corresponding to naïve wild-type, naïve IFNA Receptor knock-out, infected wild-type, and IFNA Receptor knock-out mice. Differentially-expressed genes of microglia from the two groups of infected mice are documented. Gene set enrichment analysis showing the top 500 genes assigned to Reactome pathways from infected IFNA Receptor knock-out versus naïve, and infected WT versus naïve has been generated. These data will be useful for those interested in microglia cells, and in experimental cerebral malaria.
\end{abstract}

Keywords: Malaria, Microglia, Plasmodium berghei, Experimental cerebral malaria, Type I Interferons, Microarray

\section{Objective}

Cerebral Malaria is one of the most severe complications of infection with the malaria parasite Plasmodium falciparum. A widely used animal model to investigate pathogenic processes in cerebral malaria, and the contribution of the host response, is the C57BL/6 mouse infected with the ANKA strain of Plasmodium berghei (experimental cerebral malaria, or ECM, [1]). We showed previously that microglia are activated in the brains of mice infected

\footnotetext{
*Correspondence: jean.langhorne@crick.ac.uk

${ }^{\dagger}$ Carlos Talavera-López and Barbara Capuccini are contributed equally to this work

${ }^{1}$ Malaria Immunology Laboratory, The Francis Crick Institute, 1 Midland Road, London NW1 1AT, UK

Full list of author information is available at the end of the article
}

with $P$. berghei ANKA undergoing ECM. At the time of ECM symptoms, immune response and chemokine genes were significantly upregulated. Gene Ontology analysis and functional gene enrichment suggested that these responses were driven by Type I interferons [2]. In support of this, we showed that IFN $\beta$ activated microglia in vitro to produce those chemokines, whose gene expression was upregulated in the microarray analysis [2]. As Type 1 IFN signaling can have different roles in malaria infections it would be important to determine the contributions of signaling through the Type I IFN receptor on microglia in ECM, and thus whether microglia play any part on the pathogenesis, or control of pathology, in ECM, which might have implications for human disease. We wanted to investigate the possible effects on microglia and ECM of abrogating signaling through the 
IFN-1 receptor. In the analysis shown in this Data Note, we compared the transcriptome of purified microglia from $P$. berghei-infected IFNA Receptor knock-out mice with that of infected wild-type C57BL/6 mice using Illumina Beadarrays.

An analysis of gene expression microglia in wild-type C57Bl/6 mice has been published [2], but a comparative study with microglia from IFNA Receptor knock-out mice has not been documented previously. These microarray results have not been incorporated into a research publication as we could not continue this study in this form as the Illumina Beadarrays have been discontinued. Nevertheless, we believe these data may be a useful for those research groups interested in activation of microglia in various settings and for malaria immunologists interested in the mechanisms of ECM in this rodent model.

\section{Data description}

We collected gene expression data from microglia isolated from wild-type C57Bl/6 mice and IFNA Receptor knock-out mice infected with Plasmodium berghei ANKA using Illumina Beadarrays (Table 1, data file 1).

Female C57BL/6 mice and mice lacking the IFNA Receptor (IFNARKO) (aged 6-9 weeks of age) were obtained from the SPF breeding unit of the Mill Hill Laboratory, Francis Crick Institute, and for experimental work were conventionally housed with sterile bedding, food and irradiated water. Room temperature was $22{ }^{\circ} \mathrm{C}$ with a $12 \mathrm{~h} \mathrm{light/dark} \mathrm{cycle;} \mathrm{food} \mathrm{and} \mathrm{water} \mathrm{were}$ provided ad libitum. Mice were injected with $10^{5} \mathrm{P}$. berghei ANKA infected erythrocytes intraperitoneally. Mortality, parasitemia and clinical scores indicative of ECM were monitored daily. Naïve and day 7-infected (d7) infected WT and IFNARKO mice were euthanised using pentobarbital, injected intraperitoneally $(600 \mathrm{mg} /$ $\mathrm{kg}$ body weight). Isolation of microglia is described in detail in [2]. Briefly, microglia were isolated from the brains of uninfected $\mathrm{C} 57 \mathrm{Bl} / 6$ and IFNARKO mice and from both groups of infected mice. Microglia (CD451 ${ }^{\text {ow }}$ and $C D 11 b^{+}$) were purified from other brain cells by flow cytometry (MoFlo XPD, Beckman Coulter) using a combination of fluorophore conjugated antibodies: APC-anti-CD11b, PE-CD45, APCCy7-Ly6C, pacific blue- $-\mathrm{H}-2 \mathrm{~Kb}$ (Biolegend). Cells were washed and resuspended in PBS containing 2\% FCS. Analysis was carried out using FlowJo-X software (Treestar). The sorted cells were confirmed as microglia based on the lack of cell surface marker Ly6C.

Total RNA was extracted immediately after sorting from approximately $10^{5}$ microglial cells using Ribopure kit (Ambion), and concentrations determined by Qubit quantitation using the HS assay kit (ThermoFisher Scientific). Quality was assessed by the Agilent 2100 Bioanalyzer; samples with a RIN score above 8.50 were used. Total RNA (300 ng) of each sample was amplified using the Total prep RNA amplification kit (Illumina) and Amplified cDNA (1500 ng) were then hybridized to Illumina MOUSE WG-6 V2.0 Beadarrays at $58{ }^{\circ} \mathrm{C}$ for $14-20 \mathrm{~h}$ at the High Throughput Screening facility of the Francis Crick institute RNA and cDNA were quantified by Qubit fluorometric quantitation and the quality were analysed using Agilent 2100 Bioanalyzer at each step ([2] and Table 1, Data file 1)

Data analysis was conducted using the limma package [3] within R v3.5.1 running Bioconductor v3.7. Illumina idat files were read using "read.idat" function together with manifest file MouseWG-6_V2_0_R3_11278593_A. bgx downloaded from the Illumina website. Detection $p$-values were calculated using the "detectionPValues" function with default settings. Background correction was performed using negative control probes followed by quantile normalization using negative and positive control probes via the "neqc" function. Normalised expression values are reported in a $\log _{2}$ scale.

Principal Component Analysis was performed on the 500 genes showing greatest variance across samples (Table 1, Data file 2).

Differential gene expression was assessed between infected and naïve cell states within $\mathrm{KO}$ and WT cells separately using a linear model (Table 1, Data files 3 and 4). Significance was determined using a threshold

Table 1 Overview of data files/data sets

\begin{tabular}{|c|c|c|c|}
\hline Label & Name of data file/data set & $\begin{array}{l}\text { File types } \\
\text { (file extension) }\end{array}$ & Data repository and identifier (DOI or accession number) \\
\hline Data file 1 & Microarray data C57BI/6 mice infected with P. berghei & Illumina idat & https://www.ncbi.nlm.nih.gov/geo/query/acc.cgi?acc=GSE1 19650 \\
\hline Data file 2 & PCA.pdf & pdf & https://doi.org/10.6084/m9.figshare.7171952 \\
\hline Datafile 3 & wt.inf_vs_wt.naive-p01.fc2.results.txt & $t x t$ & https://doi.org/10.6084/m9.figshare.7171952 \\
\hline Data file 4 & ko.inf_vs_ko.naive-p01.fc2.results.txt & $t x t$ & https://doi.org/10.6084/m9.figshare.7171952 \\
\hline Data file 5 & ToppGene.enrichment.barplot.pdf & $p d f$ & https://doi.org/10.6084/m9.figshare.7171952 \\
\hline Data file 6 & ko_specific_treatment_effect.heatmap.pdf & $p d f$ & https://doi.org/10.6084/m9.figshare.7171952 \\
\hline
\end{tabular}


based on a FDR $\leq 0.01$ together with an absolute fold change $\geq 2$.

The two resulting lists were ordered by absolute fold change and the top 500 unique Entrez gene identifiers from each were put forward for gene list enrichment analysis using the ToppGene Suite [4]. Hits to the Reactome [5] pathway $(\mathrm{FDR} \leq 0.01)$ are presented in the barplot (Table 1, Data file 5).

A nested interaction formula was used to select genes responding differently to infection between $\mathrm{KO}$ and WT cells. Genes showing a KO specific response but remain unchanged in WT cells were selected for visualisation in a heatmap (Data file 6). Each gene's expression across samples was converted to a $\mathrm{z}$-score to aid visualisation. Clustering of row and columns was conducted using complete linkage on a Euclidean distance matrix.

\section{Limitations}

The sample size for each group is relatively small, 5 mice per group.

There is only one time-point in the infection.

It was intended to continue this study; however, Illumina ceased production of these arrays during the study, and therefore the experiment could not be extended using this microarray format.

\section{Abbreviations}

ECM: experimental cerebral malaria; IFNARKO: Interferon Alfa Receptor 1 knock-out; iRBC: infected red blood cells; KO: knock out; P. berghei: Plasmodium berghei; WT: wild-type.

\section{Authors' contributions}

$\mathrm{BC}$ designed the study, carried out the experimental work with mice, isolated the microglia, extracted RNA and prepared material for microarray hybridisation. JWL assisted with the experimental work. CT and RM carried out the analysis of the microarray data, and wrote the paper. JL conceived the project, and wrote the paper. All authors read and approved the final manuscript.

\section{Author details}

1 Malaria Immunology Laboratory, The Francis Crick Institute, 1 Midland Road, London NW1 1AT, UK. ${ }^{2}$ Present Address: Polo di Genomica Genetica e Biologia, Via Fiorentina 1, 53100 Siena, Italy. ${ }^{3}$ Present Address: Division of Pediatric Infectious Diseases, State Key Laboratory of Biotherapy, West China Second Hospital, Sichuan University and Collaboration Innovation Centre, Chengdu, China. ${ }^{4}$ Bioinformatics and Biostatistics team, The Francis Crick Institute, 1 Midland Road, London NW1 1AT, UK.

\section{Acknowledgements}

We thank the BRF, Advanced Sequencing, Bioinformatics and Flow Cytometry facilities at the Francis Crick Institute for excellent animal husbandry and technical support.

\section{Competing interests}

The authors declare that they have no competing interests.

\section{Availability of data materials}

The datasets generated and analysed (data file 1; data file 2; data file 3; data file 4, data file 5) during the current study are available on: https://www.ncbi. $\mathrm{nlm}$.nih.gov/geo/query/acc.cgi?acc=GSE119650 and the figshare repository: Talavera-López, Carlos; Capuccini, Varbara; Lin, Jing-wen; Mitter, Richard; Langhorne, Jean; (2018). figshare https://figshare.com/s/37fc3835ea53be4bf94f.

\section{Consent for publication}

There are no data from individual humans.

\section{Ethics approval and consent to participate}

The study was carried out in accordance with the UK Animals (Scientific Procedures) Act 1986 (Home Office licence 80/2538 and 70/8326), was approved by the MRC National Institute for Medical Research Ethical Committee and by The Francis Crick Institute Ethical Committee.

\section{Funding}

This work was supported by the Francis Crick Institute which receives its core funding from the UK Medical Research Council (FC10101), Cancer Research UK (FC001101) and Wellcome (FC001101). BC was supported by an iMove Marie Curie postdoctoral fellowship. The funding bodies had no role in the design of the study, collection, analysis, and interpretation of data or in writing the paper.

\section{Publisher's Note}

Springer Nature remains neutral with regard to jurisdictional claims in published maps and institutional affiliations.

Received: 30 September 2018 Accepted: 15 December 2018

Published online: 20 December 2018

\section{References}

1. de Oca MM, Engwerda C, Haque A. Plasmodium berghei ANKA (PbA) infection of C57BL/6J mice: a model of severe malaria. Methods Mol Biol. 2013;1031:203-13. https://doi.org/10.1007/978-1-62703-481-4_23.

2. Capuccini B, Lin J, Talavera-López C, Khan SM, Sodenkamp J, Spaccapelo $\mathrm{R}$, Langhorne J. Transcriptomic profiling of microglia reveals signatures of cell activation and immune response, during experimental cerebral malaria. Sci Rep. 2016;6:39258. https://doi.org/10.1093/nar/gkv007.

3. Ritchie ME, Phipson B, Wu D, Hu Y, Law CW, Shi W, Smyth GK. limma powers differential expression analyses for RNA-sequencing and microarray studies. Nucleic Acids Research. 2015;43(7):e47. https://doi.org/10.1093/ nar/gkv007.

4. Chen J, Bardes EE, Aronow BJ, Jegga AG. ToppGene Suite for gene list enrichment analysis and candidate gene prioritization. Nucleic Acids Res. 2009;37(Web Server issue):W305-11. https://doi.org/10.1093/nar/gkp427.

5. Fabregat A, Jupe S, Matthews L, Sidiropoulos K, Gillespie M, Garapati P, Haw R, Jassal B, Korninger F, May B, Milacic M, Roca CD, Rothfels K, Sevilla C, Shamovsky V, Shorser S, Varusai T, Viteri G, Weiser J, Wu G, Stein L, Hermjakob H, D'Eustachio P. The reactome pathway knowledgebase. Nucleic Acids Res. 2018;46(D1):D649-55. https://doi.org/10.1093/nar/gkx1132. 